\title{
Seismic Soil Liquefaction for Deterministic and Probabilistic Approach Based on in Situ Test (CPTU) Data
}

\author{
Shpresa Gashi, Neritan Shkodrani \\ Department of Civil Engineering, Polytechnic University of Tirana, Tirana, Albania \\ Email: gashi84 shpresa@yahoo.com
}

Received 4 February 2015; accepted 3 April 2015; published 7 April 2015

Copyright (C) 2015 by authors and Scientific Research Publishing Inc.

This work is licensed under the Creative Commons Attribution International License (CC BY). http://creativecommons.org/licenses/by/4.0/

(c) (i) Open Access

\begin{abstract}
One of the most devastating effects of earthquakes in the seismic regions is liquefaction. Many research works have been done in this field and at present different methods are available for the liquefaction potential assessment. The liquefaction is a very significant phenomenon in clayey silty soils, silty sands and also sands. The high potential of liquefaction is generally recognized when these type of soils are laid under the hydrostatic water table. This paper make an overview of two different methods for the evaluation of liquefaction potential, and a case study is presented. Two methods presented here are the Deterministic Approach proposed by Robertson and Wride (1998), and the Probabilistic Approach proposed by Moss and co-workers. Case study of the liquefaction potential evaluation is done for the Golem area, where geotechnical data from CPTU test were collected. The results of analysis in the Golem area show that liquefaction has medium susceptibly to occur. From the analyses, it is shown that the Probabilistic Approach gives more accurate information about the risk of liquefaction than the Deterministic Approach.
\end{abstract}

\section{Keywords}

Liquefaction Assessment, Deterministic Approach, Probabilistic Approach, CPTU Test

\section{Introduction}

Liquefaction is a phenomenon in which the strength and stiffness of a soil is reduced by earthquake shaking or other dynamic loading. Liquefaction happens when there is a loose of strength in saturated and cohesion-less soils because of increased pore water pressures and hence reduced effective stresses due to dynamic loading. Liquefaction has been responsible for tremendous amount of damage in historical earthquake around the world. 
Common examples of liquefaction-induced damages includes, tilting or overturning of buildings, flow failure of steeply sloping ground such as dams and lateral spreading of softly to moderately sloping ground.

One of the first and the most widely used methods to quantify the liquefaction resistance of the soils is the simplified procedure developed by Seed \& Idriss (1971) and later by other authors as Robertson P.K (2010). In the deterministic approach, the value of a hazard parameter of interest is estimated for a specified earthquake magnitude assumed to occur at a fixed sources-to-site distance (e.g., Reiter, 1990; Anderson, 1997; Krinitzsky, 2002). Varies models for estimating the probability of liquefaction have been proposed (Liao et al., 1988; Juang et al., 2000, 2002, 2003; Cetin et al., 2004; Idriss \& Boulanger 2004; Moss 2003). The evaluation of liquefaction involves two stages: 1) evaluation of earthquake loading and 2) evaluation of soils strength against earthquake loading. The earthquake loading in soil is expressed using the term Cyclic Stress Ratio (CSR) and the soil strength to resist liquefaction is expressed using the term Cyclic Resistance Ratio (CRR). For the deterministic approach to evaluate the liquefaction potential is used the Factor of Safety $(F S)$. According to the values of the $F S$ is accepted that liquefaction has high susceptibly to happen if $F S<1$, has medium susceptibly if $1.0<F S<$ 1.25 and low susceptibly when $F S>1.25$. For the probabilistic approach to evaluate liquefaction potential is used the Probability of Liquefaction (PL). According to the values of PL, for values in the interval $0.85 \leq \mathrm{PL}<1$ liquefaction is almost certain; in the interval $0.65 \leq \mathrm{PL}<0.85$ it is probable; in the interval $0.35 \leq \mathrm{PL}<0.55$ it is uncertain; in the interval $0.5 \leq \mathrm{PL}<0.35$ it is unlikely and in the interval $0.0 \leq \mathrm{PL}<0.15$ liquefaction does not occur. This paper represents an overview of two different liquefaction potential evaluation methods which are currently in use. A case study based on these two methods is presented here.

\section{Liquefaction Susceptibility Based on in-Situ Methods}

The evaluation of liquefaction potential is developed along two lines: One is by means of laboratory testing (e.g., cyclic triaxle test and cyclic simple shear test) of undisturbed samples, and the other involves using the empirical correlation available with various in situ tests such as Standard Penetration Test (SPT), Cone Penetration Test (CPT), shear wave velocity measurement (Vs) and the Becker Penetration Test (BPT). Because of the highquality testing of granular soils, the use of in-situ tests along with the case histories-calibrated empirical relationships (i.e. liquefaction boundary curves) has been, and is still, the dominant approach in engineering practice.

\subsection{Evaluation of Liquefaction Potential Based in CPTU Data for the Deterministic Approach}

The "simplified procedure" originally developed by Seed and Idriss (1971) [1] and later by other author as Robertson P.K (2010) [2] is used to evaluate liquefaction potential. This procedure essentially compares the cyclic resistance ration $(C R R)$ at a given depth with the earthquake-induced cyclic stress ration $(C S R)$ at the depth from a specified design earthquake.

\subsubsection{Evaluation of Cyclic Stress Ratio (CSR)}

The average uniform cyclic stress ratio (CSR) within a liquefiable layer is given by Seed \& Idriss (1971).

$$
\operatorname{CSR}_{7.5}=\frac{\tau_{c y c}}{\sigma_{v 0}^{\prime}}=0.65 \cdot\left(\frac{a_{\max }}{g}\right) \cdot\left(\frac{\sigma_{v 0}}{\sigma_{v 0}^{\prime}}\right) \cdot\left(\frac{r_{d}}{M S F}\right)
$$

where:

$a_{\max }=$ peak horizontal ground acceleration generated by the earthquake; $g$ = acceleration of gravity;

$\sigma_{v 0}=$ initial vertical total stress; $\sigma_{v 0}^{\prime}=$ initial vertical effective stress; $r_{d}=$ stress reduction factor; $M S F=$ magnitude scaling factor.

Liao and Whitman [3] proposed the calculation of $r_{d}$ as a function only of the soil depth as follows:

$$
\begin{array}{cc}
r_{d}=1.0-0.00765 \cdot z & \text { if } z<9.15 \mathrm{~m} \\
r_{d}=1.174-0.0267 \cdot z & \text { if } z \geq 9.15 \mathrm{~m}
\end{array}
$$

where: $z=$ is the depth in meters.

The CSR for a magnitude different from 7.5 can be calculated as follows 


$$
\operatorname{CSR}_{M}=\operatorname{CSR}_{M=7.5} \cdot \mathrm{MSF}
$$

\subsubsection{Magnitude Scaling Factor, MSF}

The magnitude scaling factor, MSF, has been used to adjust the induced CSR during an earthquake of magnitude $M_{w}$ by using the $C S R$ for an earthquake magnitude, $M_{w}=7.5$. The MSF is thus defined as:

$$
M S F=C S R_{M_{w}} / C S R_{M_{w}=7.5}
$$

Thus, MSF provides an approximate representation of the effects of shaking duration or equivalent number of stress cycles. Values of magnitude scaling factors are derived by combining: 1) Correlation of the number of equivalent uniform cycles versus earthquake magnitude, and 2) Laboratory-based relationships between the cyclic stress ratios required to cause liquefaction and the number of uniform stress cycles. The value of scaling factor, MSF, is proposed by various researchers (reproduced from Youd and Nobel 1997) [4].

In this paper, magnitude scaling factor, MSF, proposed by Idriss (1990) [5] is used to evaluate the liquefaction potential:

$$
M S F=10^{2.24} / M_{w}^{2.56}
$$

\subsubsection{Evaluation of Cyclic Resistance Ratio (CRR)}

The $C R R$ is evaluated by using the CPTU test, which is considered to be a reliable test for soil investigation by today's standards, providing important information on soil type and geotechnical parameters.

For the cyclic resistance ratio of clean sands and a magnitude of $7.5\left(C R R_{7.5}\right)$, Robertson and Wride (1998, 2004, and 2010) [6] have proposed the following equations:

$$
\begin{array}{cc}
C R R_{c}=0.833 \cdot\left[\left(q_{c 1, N}\right)_{c s} / 1000\right]+0.05 & \text { for }\left(q_{c 1, N}\right)_{c s}<50 \\
C R R_{c}=93 \cdot\left[\left(q_{c 1, N}\right)_{c s} / 1000\right]^{3}+0.08 & \text { for } 50 \leq\left(q_{c 1, N}\right)_{c s} \leq 160
\end{array}
$$

where:

$\left(q_{c 1, N}\right)_{c s}=$ is the normalized cone penetration resistance, corrected for the fine content influence

$$
\left(q_{c 1, N}\right)_{c s}=K_{c} \cdot\left(q_{c 1, N}\right)
$$

" $K_{c}$ " = is a correction factor that is a function of grain size characteristics (combined influence of fines content and plasticity) of the soil.

$$
\begin{gathered}
K_{c}=1 \quad \text { for } I_{c} \leq 1.64=K_{c} \cdot\left(q_{c 1, N}\right) \\
K_{c}=-0.403 \cdot I_{c}^{4}+5.581 \cdot I_{c}^{3}-21.631 \cdot I_{c}^{2}+33.75 \cdot I_{c}-17.88 \quad \text { for } I_{c} \geq 1.64
\end{gathered}
$$

Robertson and Wride, (1988) suggested estimating the grain size characteristics using the soil behaviour chart by Robertson (1990) [7] and the soil behaviour type index, $I_{c}$.

where,

$$
I_{c}=\left[(3.47-\log Q)^{2}+(1.22+\log F)^{2}\right]^{0.5}
$$

and

$$
\begin{aligned}
& Q=\frac{q_{c}-\sigma_{v}}{P_{a}} \cdot\left(\frac{P_{a}}{\sigma_{v}^{\prime}}\right)^{n} \\
& F_{R}=100 \cdot \frac{f_{s}}{\left(q_{c}-\sigma_{v}\right)}
\end{aligned}
$$

where:

$Q=$ is the normalized cone penetration resistance; $F_{R}=$ is normalized friction ratio; $\sigma_{v}$ and $\sigma_{v}^{\prime}=$ are the ini- 
tial total and effective overburden stresses, respectively; $P_{a}=$ is the atmospheric pressure; $q_{c}=$ is the measured tip resistance; $f_{s}=$ is the CPT sleeve friction resistance; $n=$ is the stress exponent.

The stress exponent " $n$ " varies according to the soil type. The typical value of " $n$ " is 0.5 for clean sands and 1 for clays. For silts and silty sand an intermediate value between 0.5 and 1 is appropriate.

The normalized cone penetration resistance " $Q$ " is calculated first, assuming that $n=1$. The soil behaviour type index, $I_{c}$ calculated for $n=1$ is than introduced in the next step of calculation of ' $n$ ' value:

$$
n=0.381 \cdot\left(I_{c}\right)+0.05 \cdot\left(\frac{\sigma_{v}^{\prime}}{P_{a}}\right)-0.15
$$

Then, a new " $Q$ " value is calculated with the last value of " $n$ "; an iteration procedure through " $I_{c}$ " and " $Q$ " proposed by Robertson (1990) is used to evaluate " $n$ " until the difference between the last values of " $n$ " is less than 0.01 .

The last found value of " $n$ " allows to calculate $q_{c 1, N}$, in Equation (5) which is the normalized cone penetration resistance defined as follow:

$$
q_{c 1, N}=\frac{q_{c}-\sigma_{v}}{P_{a 1}} \cdot\left(\frac{P_{a}}{\sigma_{v}^{\prime}}\right)^{n}
$$

The final value of " $I_{c}$ " is used to compute the value of $K_{c}$ given in Equations (6a) and (6b).

\subsubsection{Evaluating Factor of Safety, FS}

When the values of CRR and CSR are established for a stratum at a given depth, FS against liquefaction should be calculated. The FS against liquefaction is defined as (Coduto, 2003) [8] According to the values of the Factor of Safety is accepted that liquefaction has high susceptibly to happen for $F S<1$, has medium susceptibly for $1.0<$ $F S<1.25$ and low susceptibly for $F S>1.25$.

$$
F S=C R R / C S R
$$

\subsection{Evaluation of Liquefaction Potential Based in CPTU Data for the Probabilistic Approach.}

Various models for estimating the probability of liquefaction have been proposed (Liao et al., 1988; Juang et al., 2000, 2002; Cetin et al., 2004, Moss 2006). Here, we are going to present the model proposed by Moss (2006).

\subsubsection{Evaluation of Cyclic Stress Ratio (CSR)}

Equation (1) is used to calculate cyclic stress ratio (CSR). There are two differences: one is that the reduction factor of the stress is calculated according to Cetin and Seed (2004) [9] that proposed the calculation of $r_{d}$ as a function of the soil depth (d), the earthquake magnitude $\left(M_{w}\right)$ and $a_{\max }$ as follows:

For depth $(d)<20 \mathrm{~m}$

$$
r_{d}=\frac{\left[1+\frac{-9.147-4.173 \cdot a_{\max }+0.652 \cdot M_{w}}{10.567+0.089 \cdot \mathrm{e}^{0.089 \cdot\left(-d \cdot 3.28-7.760 \cdot a_{\max }+78.576\right)}}\right]}{\left[1+\frac{-9.147-4.173 \cdot a_{\max }+0.652 \cdot M_{w}}{10.567+0.089 \cdot \mathrm{e}^{0.089 \cdot\left(-7.760 \cdot a_{\max }+78.576\right)}}\right]} \pm \sigma
$$

For depth $(d) \geq 20 \mathrm{~m}$

$$
r_{d}=\frac{\left[1+\frac{-9.147-4.173 \cdot a_{\max }+0.652 \cdot M_{w}}{10.567+0.089 \cdot \mathrm{e}^{0.089 \cdot\left(-d 3.28-7.760 \cdot a_{\max }+78.576\right)}}\right]}{\left[1+\frac{-9.147-4.173 \cdot a_{\max }+0.652 \cdot M_{w}}{10.567+0.089 \cdot \mathrm{e}^{0.089 \cdot\left(-7.760 \cdot a_{\max }+78.576\right)}}\right]}-0.0014 \cdot(d \cdot 63.28-95) \pm \sigma
$$

where: $d=$ depth in meters at the midpoint of the critical layer; $M_{w}=$ moment magnitude. The standard deviation for $r_{d}$ is as follows:

for $d<12.2 \mathrm{~m}$ 


$$
\sigma_{r_{d}}(d)=(d \cdot 3.28)^{0.864} \cdot 0.00814
$$

for $d \geq 12.2 \mathrm{~m}$

$$
\sigma_{r_{d}}(d)=40^{0.864} \cdot 0.00814
$$

and the second one is that instead of MSF the method is using the Duration Weighting Factor, $D W F_{M}$

The duration weighting factor $\left(D W F_{M}\right)$ has previously been developed using different approaches. Cetin et al. (2004) recommended the calculation of $D W F_{M}$ as follows:

$$
D W F_{M_{w}}=17.84 \cdot M_{w}^{-143}
$$

The final values of $C S R$ used for the calculation is as follows:

$$
C S R_{M_{w}=7.5}=\frac{C S R_{M_{w}=?}}{D W F_{M_{w}}}
$$

\subsubsection{Evaluation of the Probability of Liquefactions}

The cyclic resistance ratios for a given probability of liquefaction according to Moss et al. (2006) [10] can be calculated as follows:

$$
\begin{gathered}
C R R=\exp \left[\frac{\left.\begin{array}{l}
q_{c, 1}^{1.045}+q_{c, 1}\left(0.110 \cdot R_{f}\right)+\left(0.110 \cdot R_{f}\right)+c\left(1+0.850 \cdot R_{f}\right)-0.848 \cdot \ln \left(M_{w}\right) \\
-0.002 \cdot \ln \left(\sigma_{v 0}^{\prime}\right)-20.923+1.632 \cdot \Phi^{-1}\left(P_{L}\right)
\end{array}\right]}{7.177}\right] \\
P_{L}=\Phi\left\{-\frac{\left[\begin{array}{l}
q_{c, 1}^{1.045}+q_{c, 1}\left(0.110 \cdot R_{f}\right)+\left(0.110 \cdot R_{f}\right)+c\left(1+0.850 \cdot R_{f}\right)-7.177 \cdot \ln (C S R) \\
-0.848 \cdot \ln \left(M_{w}\right)-0.002 \cdot \ln \left(\sigma_{v}^{\prime}\right)-20.923
\end{array}\right]}{1.632}\right\}
\end{gathered}
$$

where:

$\Phi\left(P_{L}\right)=$ cumulative normal distribution.

$\Phi^{-1}\left(P_{L}\right)=$ inverse cumulative normal distribution function.

where:

$$
\begin{gathered}
q_{c, 1}=C_{q} \cdot q_{c} \\
C_{q}=\left(\frac{P_{a}}{\sigma_{v 0}^{\prime}}\right)^{c}
\end{gathered}
$$

where:

$q_{c, 1}=$ normalized tip resistance (in Mega Pascal); $C_{q}=$ tip normalization factor; $q_{c}=$ raw tip resistance (in Mega Pascal); $P_{a}=$ reference stress ( 1 atmosphere $=101.325$ Kilo Pascal) in compatible units;

$\sigma_{v 0}^{\prime}=$ initial effective overburden stress; $R_{f}=$ friction ratio (in percentage); and c = normalization exponent.

The value of " $c$ " can be calculated using the iterative equation:

$$
c=f_{1} \cdot\left(\frac{R_{f}}{f_{3}}\right)^{f_{2}}
$$

where:

$$
\begin{aligned}
& f_{1}=x_{1} \cdot q_{c}^{x_{2}} ; f_{2}=-\left(y_{1} \cdot q_{c}^{y_{2}}+y_{3}\right) ; \quad f_{3}=a b s\left[\log \left(10+q_{c}\right)\right]^{z_{1}} ; \\
& x_{1}=0.78 ; \quad x_{2}=-0.33 ; \quad y_{1}=-0.32 ; \quad y_{2}=-0.35 ; z_{1}=1.21
\end{aligned}
$$




$$
R_{f}=\frac{f_{s}}{q_{c}}
$$

\section{Case Study of Golem Area, Albania}

The area under study is situated at Golem municipally of Kavaja Country, at the central Albanian coast, in Tirana Prefecture (see Figure 1, at center). This is an overpopulated area and many buildings are constructed very close to the sea coast line. Generally, the buildings have shallow foundations embedded to the upper soil layers, usually at a depth of 2.0 to $4.0 \mathrm{~m}$ depth. In order to conduct this research study, 10 CPTU tests are carried out by in Situ Balkans Company in different location across the coastal line, mentioned below as boreholes, BH-i. From 10 CPTU tests, 6 of them are considered valid, which are BH-1, 2, 3, 5 and 8, as shown in Figure 1 (at left). The penetration depths varies from $8.0 \mathrm{~m}$ to $20.0 \mathrm{~m}$ The soil behavior type index $I_{c}$ is used to identify the layers with high potential of liquefaction from the CPTU test data and the detailed $I_{c}$ profile for three boreholes which are considered are showed in Figure 2. The main types of soils met in this area are SANDS and silty SANDS, silty SANDS and sandy SILT, clay and silty CLAY. From 10 CPTU tests carried out, only three of them are taken into consideration, $\mathrm{BH}-1, \mathrm{BH}-2$ and $\mathrm{BH}-8$. During the liquefaction analysis natural groundwater level is fixed at a depth of $1.7 \mathrm{~m}$ for $\mathrm{BH}-1, \mathrm{BH}-2$ and for $\mathrm{BH}-8$ natural groundwater level is fixed at $1.2 \mathrm{~m}$. From the seismic point of view this area is located in the Periardiatic Depression, denoted as PL-zone (see Figure 1, at right), strongly affected by post-Pliocene compression movements, in direct convergence with Adria microplate. It is characterized by a high seismic activity and according to the seismic hazard map of Albania for the second level of hazard that represent an earthquake with $10 \%$ probability of exceedance during the life-span of the structure (considered as 475 years) or a Peak Ground Acceleration (PGA) equal to $0.273 \mathrm{~g}$ [11]. According to the Albanian earthquakes catalogue, the expected earthquake surface wave magnitudes $\left(\mathrm{M}_{\mathrm{s}}\right)$ of the considered area vary from 4.5 - 6.6 [12]. The highest magnitude is $\mathrm{M}_{\mathrm{s}}=6.6$ (year 346 and coordinates P 41.30; L 19.30).

\section{Results}

The liquefaction potential evaluation for the deterministic method is done by using the values of Factor of Safety $(F S)$ which is given as the ratio between $C R R$ and CSR. According to the values of the Factor of Safety is accepted that liquefaction has high susceptibly to happen for $F S<1$, has medium susceptibly for $1.0<F S<1.25$ and low susceptibly for $F S>1.25$. The FS, is evaluated for the 2nd level of hazard with a maximum estimated acceleration equal to $0.273 \mathrm{~g}$ (475 years return period) or a maximum magnitude $\mathrm{M}=6.6$. The conditional liquefaction values obtained for the second hazard level Figure 3 indicate that generally the liquefaction has medium susceptibly to occur (see values between the interrupted and continuous lines). It is shown that, at BH-1 it

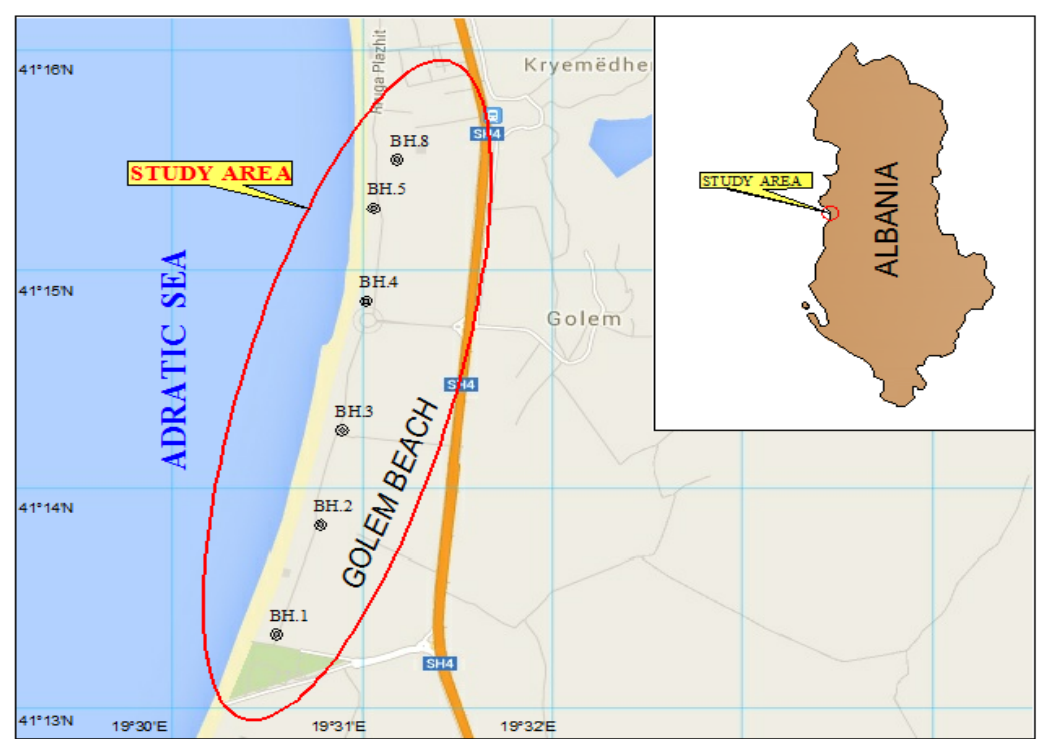

Figure 1. Geografic location of the study area. 


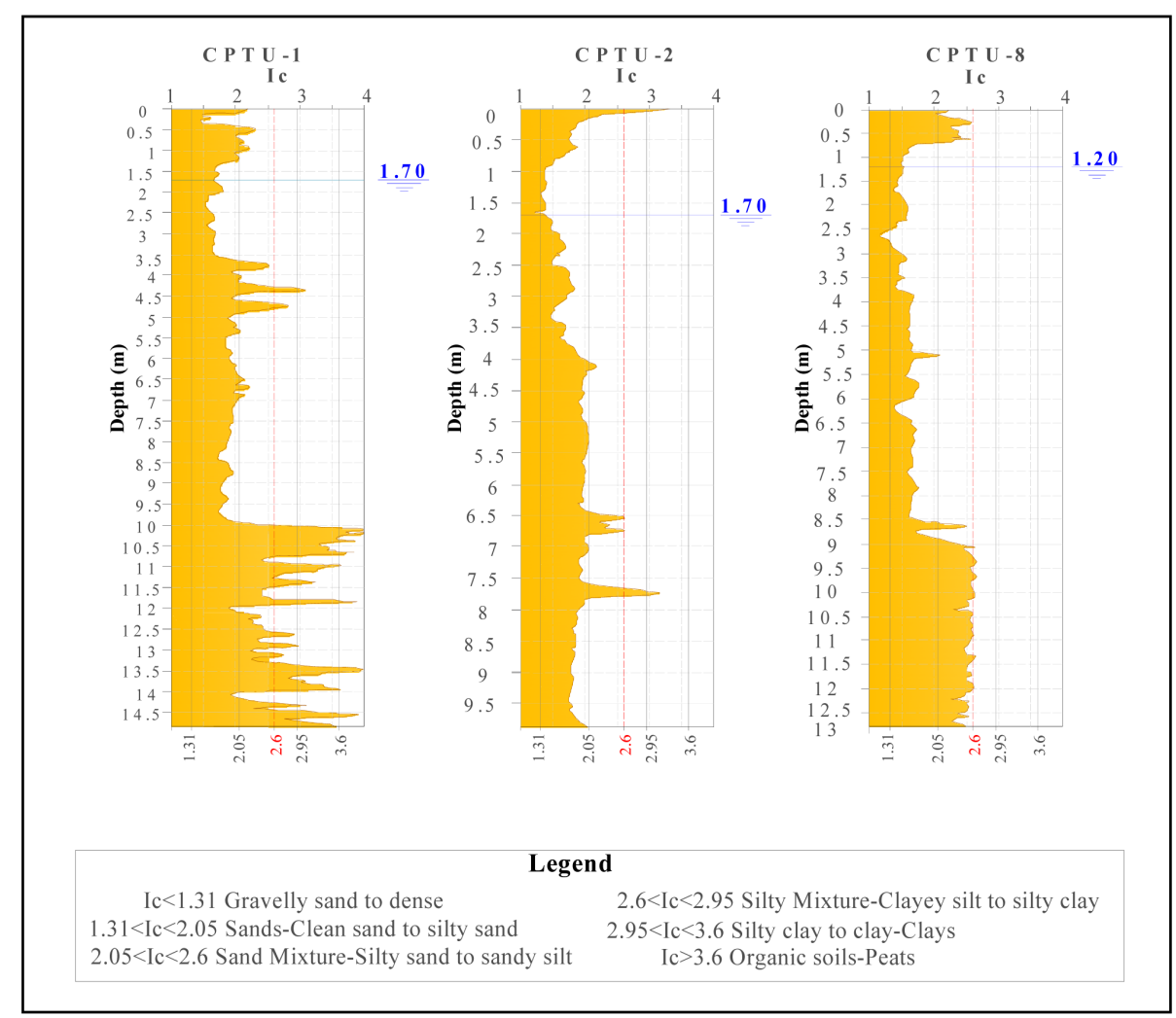

Figure 2. Detailed $I_{c}$ profile for boroholes BH-1; BH-2 and BH-8.

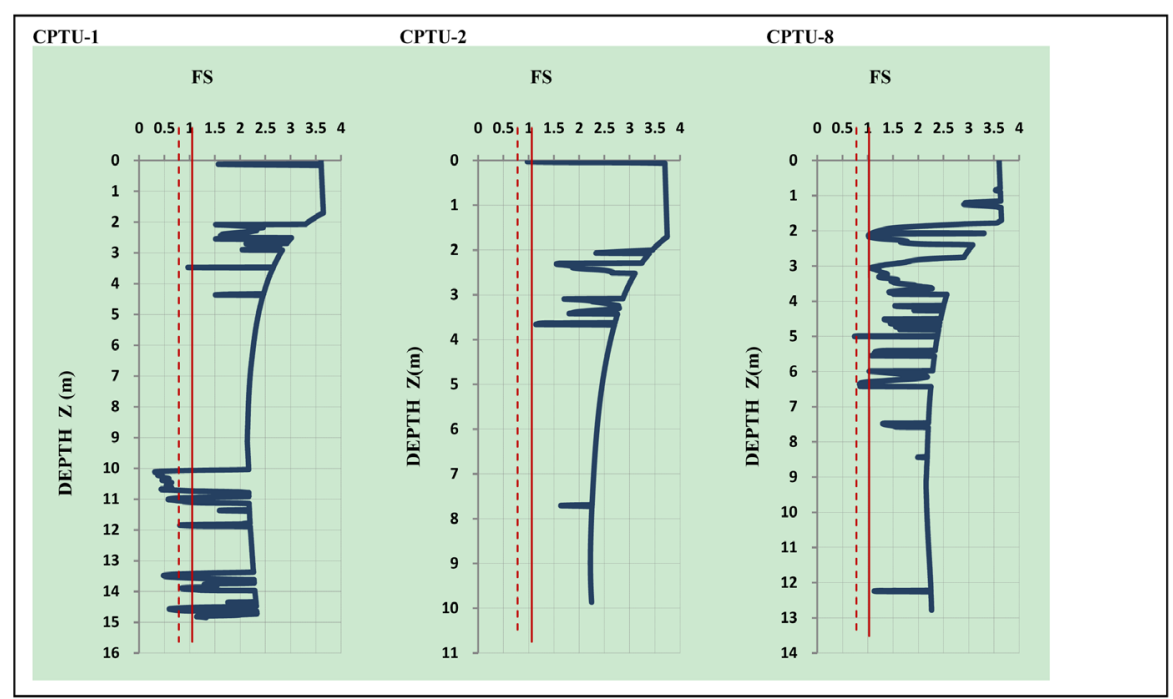

Figure 3. Evalution of Factor of Safety $(F S)$ for the deterministic methods for boreholes $\mathrm{BH}-1$; $\mathrm{BH}-2$ and $\mathrm{BH}-8$.

is a high susceptibly to liquefaction at a depth interval of $10-14.5 \mathrm{~m}$ at the case where G.W.L is accepeted at $-1.7 \mathrm{~m}$ below the ground surface and it is increased at the case where G.W.L is accepted to be $+1.0 \mathrm{~m}$ above the surface. Similar results are found for the $\mathrm{BH}-2$ and $\mathrm{BH}-8$.

The probabilistic approach is using the Probability of Liquefaction (PL) to evaluate liquefaction potential. According to the values of the PL, the liquefaction phenomenon is probable to occur, for the interval $0.85 \leq \mathrm{PL}<1$ (in this interval it is accepted that liquefaction is almost certain). For the BH-1 (see Figure 4), there are to inter- 
vals ((3.5 - 7.0) $\mathrm{m}$ and $(10.0 \mathrm{~m}-15.0) \mathrm{m})$, where probability of liquefaction is almost certain. For the BH-2 the depth of the almost certain liquefaction varies from $6.5 \mathrm{~m}$ to $8.5 \mathrm{~m}$ and for the BH-8 the depth of liquefiable layers is larger and it varies from $8.5 \mathrm{~m}$ to $12 \mathrm{~m}$ depth.

In Figure 5, the comparison between two methods is also given, and it is shown that they have almost the same results (where FS $<1$ the PL is almost 1). Between these two methods, the probabilistic methods give more accurate information about the risk of liquefaction, because gives more detailing the intervals where liquefaction

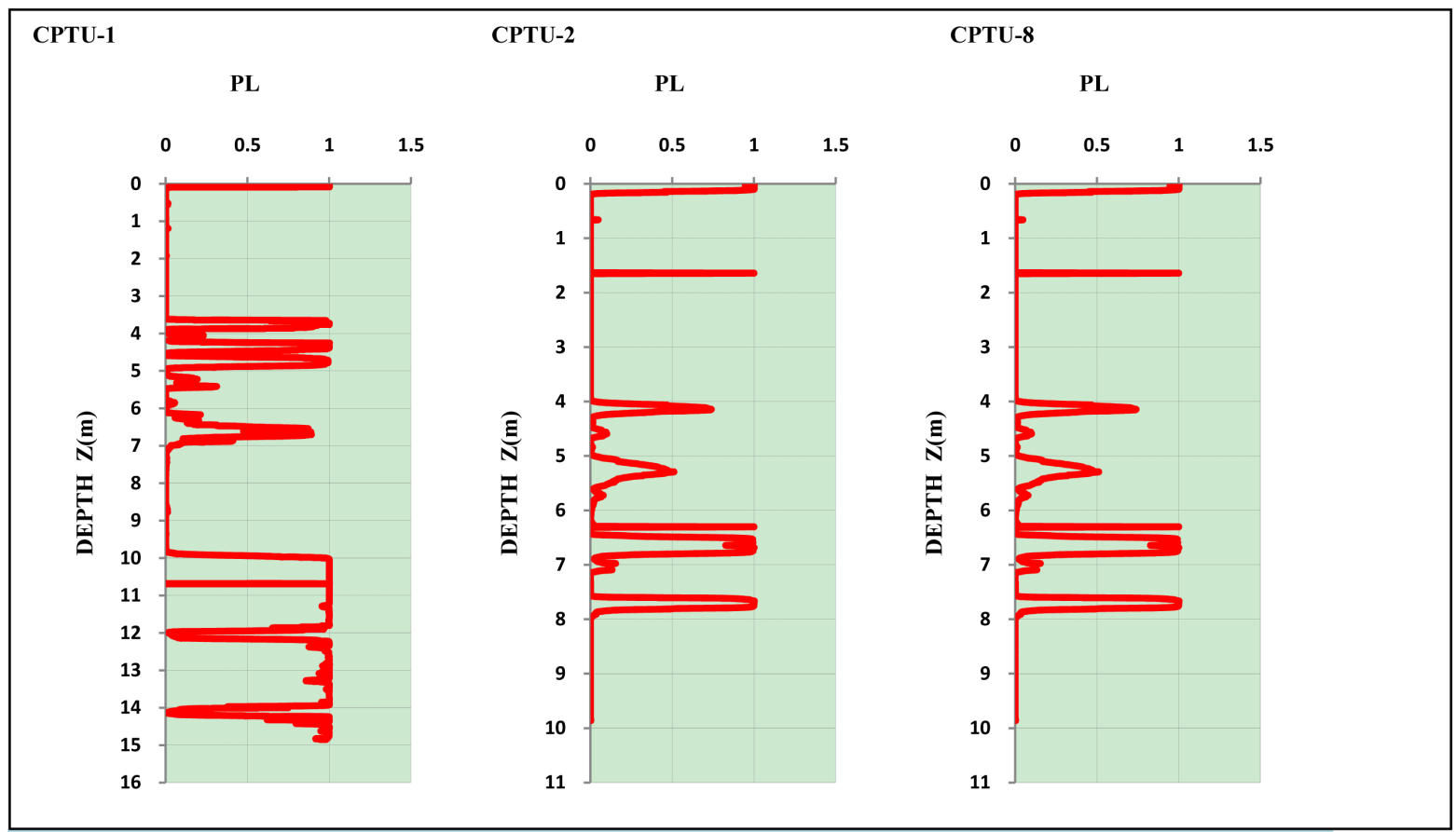

Figure 4. Evaluation of Probability of Liquefaction (PL) for the probabilistic methods for boreholes BH-1; BH-2 and BH-8.

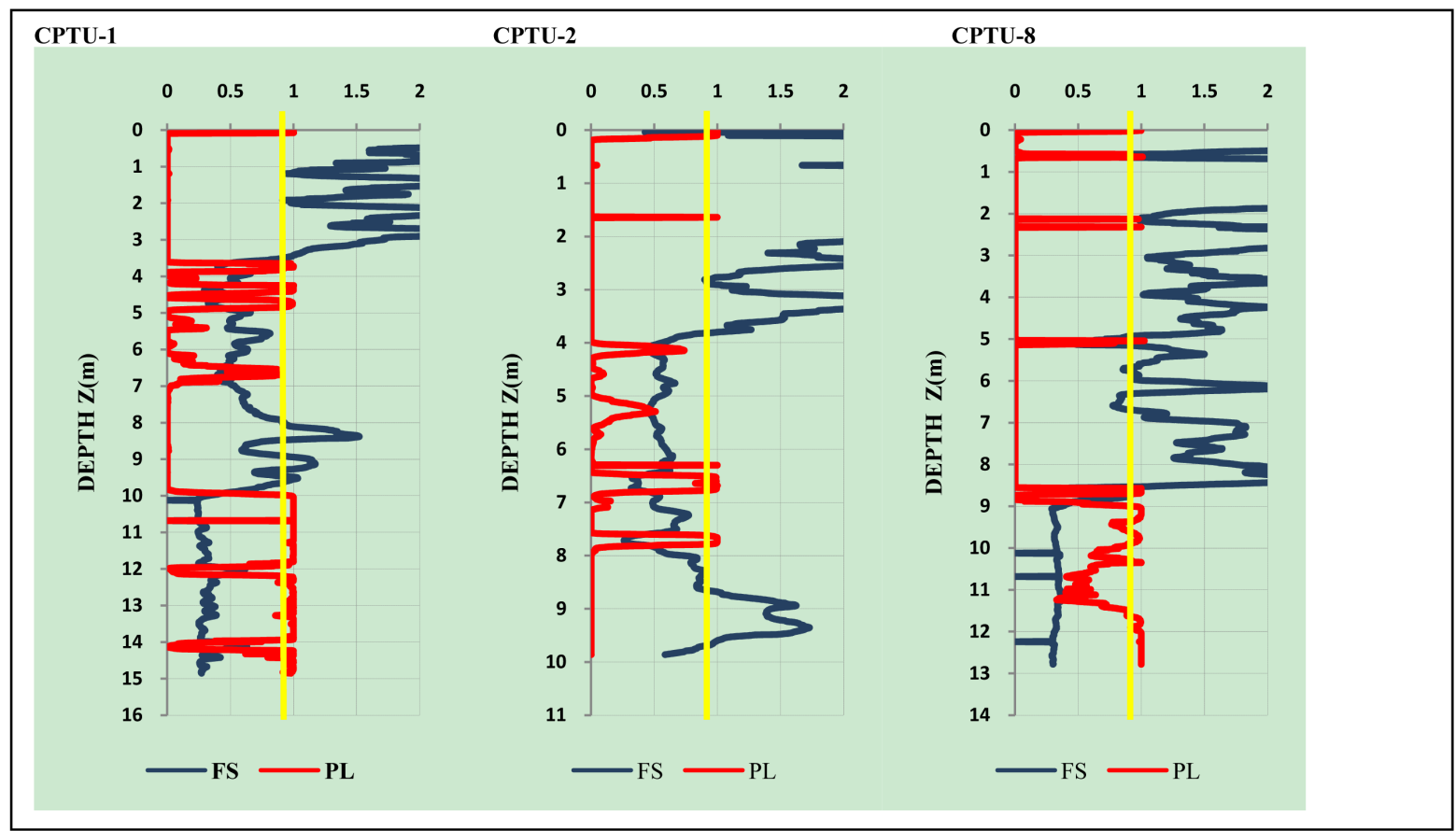

Figure 5. Comparison between Factor of Safety (FS) and probability of liquefaction (PL) for BH-1; BH-2 and BH-8. 
can happen.

\section{References}

[1] Seed, H.B. and Idriss, I.M. (1971) Simplified Procedures for Evaluating Soil Liquefaction Potential. Journal of Geotechnical Engineering, 97, 1249-1273.

[2] Robertson, P.K. (2010) Evaluation of flow liquefaction and liquefied strength using the Cone Penetration Test. Journal of Geotechnical \& Geoenvironmental Engineering, 136, 842-853. http://dx.doi.org/10.1061/(ASCE)GT.1943-5606.0000286

[3] Liao, S.S.C. and Whitman, R.V. (1986) Catalouge of Liquefaction and Non-Liquefaction Occurrences during Earthquakes. Report, Department of Civil Engineering, MIT, Cambridge.

[4] Youd, T.L. and Noble, S.K. (1997) Magnitude Scaling Factors. NCEER Workshop on Evaluation of Liquefaction Resistance of Soils, National Center for Earthquake Engineering Research, State University of New York at Buffalo, 149165.

[5] Idriss, I.M. (1990) Response of Soft Soil Sites during Earthquakes. Proc., H. Bolton Seed Memorial Symp., Vol. 2, BiTech Publishers, Vancouver, 273-290.

[6] Robertson, P.K. and Wride, C. (1998) Evaluating Cyclic Liquefaction Potential Using the Cone Penetration Test. Canadian Geotechnical Journal, 35, 442-459. http://dx.doi.org/10.1139/t98-017

[7] Robertson, P.K. (1990) Soil Classification Using the Cone Penetration Test. Canadian Geotechnical Journal, 27, 151-158. http://dx.doi.org/10.1139/t90-014

[8] Coduto, D.P. (2003) Geotechnical Engineering Principles and Practices. Chapter 3, Chapter 20.

[9] Cetin, K.O. and Seed, R.B. (2004) Nonlinear Shear Mass Participation Factor $\left(r_{d}\right)$ for Cyclic Shear Stress Ratio Evaluation. Soil Dynamics and Earthquake Engineering, 24, 103-113.

[10] Moss, R.E., Seed, R.B., Kayne, R.E., Stewart, J.P. and Kiureghian, A.D. (2006) CPT-Based Probabilistic and Deterministic Assessment of in Situ seismic Soil Liquefaction Potential. Journal of Geotechnical and Geoenvironmental Engineering, 132, 1032-1051.

[11] Sulstarova, E., Kociaj, S. and Aliaj, S.H. (1980) Seismic Regionalization of PSR of Albania. Publication of Academy of Sciences, Tirana.

[12] Aliaj, Sh., Kociaj, S., Muço, B. and Sulstarova, Sh. (2010) Seismic Regionalization of PSR of Albania. Publication of Academy of Sciences, Tirana, 221. 